Yingchao Han

Shipu Li

Xinyu Wang

Xianying Cao

Li Jia

Jianhua Li

\title{
Preparation and characterization of calcium phosphate-albumin colloidal particles by high ultrasonic irradiation
}

\section{Colloid Polym Sci (2005). DOI 10.1007/s00396-005-1317-2}

The online version of the original article can be found at http://dx.doi.org/10.1007/ s00396-005-1317-2

Y. Han $\cdot$ S. Li $(\bowtie) \cdot$ X. Wang $\cdot$ X. Cao L. Jia $\cdot$ J. Li

Biomedical Materials and Engneering Center, Wuhan university of technology, Wuhan 430070, People's Republic of China E-mail: zlhyc@yahoo.com

Tel.: + 86-27-87651852

Fax: + 86-27-87880734
In the paper " Preparation and characterization of calcium phosphate-albumin colloidal particles by high ultrasonic irradiation" by Yingchao Han, Shipu Li, Xinyu Wang, Xianying Cao, Li Jia, Jianhua Li (Colloid Polym. Sci. (2005) DOI 10.1007/s00396-005-1317-2), the following correction should be made:
The value of $7.473 \mathrm{mmol} / \mathrm{L}$ in the "Preparation of $\mathrm{CaP} / \mathrm{BSA}$ colloidal particles of Experimental" paragraph should be changed to $8.048 \mathrm{mmol} / \mathrm{L}$. 